\title{
$r$-Process Nucleosynthesis in Black Hole - Neutron Star Mergers
}

\author{
Rebecca Surman* \\ Department of Physics and Astronomy, Union College, Schenectady, NY 12308 \\ E-mail: surmanr@union.edu
}

\section{G. C. McLaughlin}

Department of Physics, North Carolina State University, Raleigh, NC 27695

\section{Ruffert}

School of Mathematics, University of Edinburgh, Edinburgh EH9 3JZ

\section{H.-Th. Janka}

Max-Planck-Institut für Astrophysik, Postfach 1317, 85741 Garching, Germany

\section{W. R. Hix}

Physics Division, Oak Ridge National Laboratory, Oak Ridge, TN 37831

\begin{abstract}
Compact object mergers have long been speculated to be a possible site of $r$-process nucleosynthesis. While most attention has been focused on the cold decompression of neutron star matter ejected from the merger, other sites within the merger likely contribute to its nucleosynthetic output. Here we consider hot outflows from the accretion disk that forms around the black hole following a black hole - neutron star merger. We begin with the results of a three-dimensional numerical merger model and carefully calculate the neutrino and antineutrino fluxes emitted from the accretion disk. We find that neutrino interactions on free nucleons in the outflowing material result in neutron excesses such that at least a weak $r$-process is produced and in some cases a main $r$-process as well. Additionally, we find that the weak $r$-process pattern calculated for certain trajectories compares favorably to the pattern observed in a weak $r$-process-enhanced halo star.
\end{abstract}

10th Symposium on Nuclei in the Cosmos

July 27 - August 1, 2008

Mackinac Island, Michigan, USA

\footnotetext{
*Speaker.
} 


\section{Introduction}

About half of the heavy elements in the solar system are known to have been produced via rapid neutron capture in the $r$-process of nucleosythesis [1,2], though where in the galaxy the $r$ process occurs is still not resolved. Many possibilities have been suggested, including, for example, core collapse supernova, e.g. [3, 4, 5, 6], neutron star mergers, e.g. [7, 8], gamma ray bursts, e.g. [9], and shocked surface layers of O-Ne-Mg cores, e.g. [10, 11], but a conclusive determination of the site has yet to be agreed upon.

Here we consider a potential astrophysical site within a black hole - neutron star (BH-NS) merger. Most calculations of $r$-process nucleosynthesis from compact object mergers have focused on the cold $[8,12]$ or mildly heated [7] neutron-rich material originating from the tidal tails that form during the merger. We focus here on a distinctly different site - that of the hot outflows from the accretion disk produced by the BH-NS merger [13]. The merger accretion disk is expected to be hot, dense, and a copious emitter of neutrinos. Any outflowing material from such a disk will be exposed to this neutrino flux. Here we examine the possibility that the neutrino interactions in this material cause it to become neutron rich and therefore an attractive potential site for $r$-process nucleosynthesis.

\section{Merger and Outflow Model}

We begin with the results of a three-dimensional black hole - neutron star merger model calculated by M. Ruffert and H.-Th. Janka and described in [13]. The model is evolved until the remains of the neutron star form an accretion disk around the black hole. We then use the temperature, density, and electron fraction of the resulting accretion disk to calculate where neutrinos and antineutrinos decouple vertically from the disk, as in [14]. We take the emitted neutrino fluxes to be thermal, with temperatures equal to the local disk temperature at the point of decoupling. The temperatures of the electron neutrinos $\left(T_{v_{e}}\right)$ and antineutrinos $\left(T_{\bar{v}_{e}}\right)$ are shown in Fig. 1. While the electron neutrinos are trapped over a larger area of the disk, the antineutrinos decouple from deeper within the disk and therefore have higher temperatures. The neutrino fluxes at every point above the disk are then calculated by integrating over the entire disk. Sample neutrino fluxes for a point above the disk are shown in Fig. 2. The net antineutrino flux at this point is both larger and hotter than the neutrino flux.

We follow the material as it outflows the disk using a parameterized wind model as in [15]. We take the outflow to be adiabatic, with velocity $v$ as a function of radius from the black hole $r$ to be $v_{\infty}\left(1-R_{0} / r\right)^{\beta}$, where $R_{0}$ is the initial distance from the black hole, $v_{\infty}$ is the final coasting speed of $0.1 c$, and $\beta$ determines how rapidly the material accelerates, with small values of $\beta$ corresponding to the most rapid acceleration. We vary the starting radius $R_{0}$ of the outflow, the entropy $s / k$ of the outflow material, and the acceleration parameter $\beta$. We calculate the nucleosynthetic outcome for each trajectory as in [9]. In addition, since the disk is not symmetric, we average the nucleosynthesis results from eight equally-spaced azimuthal angles about the disk for each $\left(R_{0}\right.$, $s / k, \beta)$. 


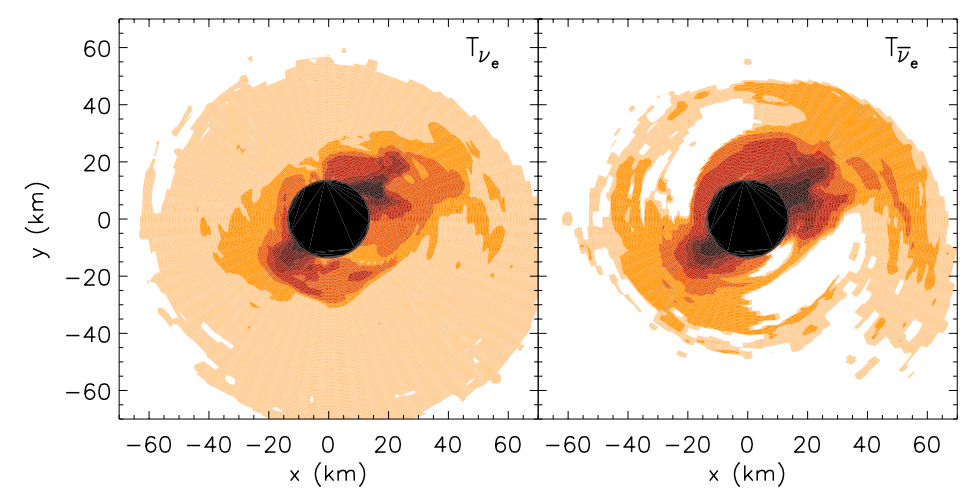

Figure 1: Plots of the electron neutrino (left panel) and electron antineutrino (right panel) temperature from the surface of the disk. Only regions where the neutrinos are trapped are shaded. Contours indicate regions, from lightest to darkest, of temperatures of $1 \mathrm{MeV}, 3 \mathrm{MeV}, 5 \mathrm{MeV}, 7 \mathrm{MeV}, 9 \mathrm{MeV}, 11 \mathrm{MeV}$ and $13 \mathrm{MeV}$. The dark center indicates the inner boundary of the numerical merger model.

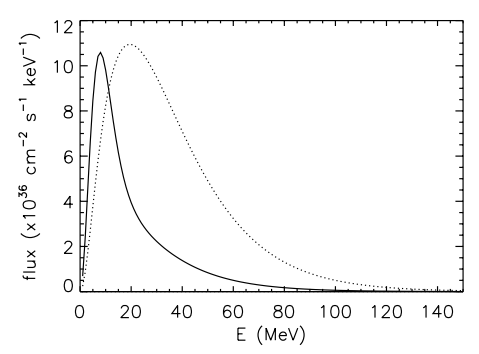

Figure 2: Shows the net electron neutrino (solid line) and electron antineutrino (dashed line) fluxes at a point $133 \mathrm{~km}$ above the plane of the disk and $295 \mathrm{~km}$ from the black hole.

\section{Results}

The left panel of Fig. 3 shows the angle-averaged results from two trajectories representative of our calculated nucleosynthetic outcomes. In all cases we see the production of neutron-rich nuclei. The degree to which a successful $r$-process is obtained is determined by the neutron-to-proton ratio in the outflow, which is set by weak interactions above the disk. As the material leaves the disk, electron and positron capture dominate at first, then, as the temperatures of the electrons and positrons drop below that of the neutrinos, neutrino interactions take over. Since the antineutrinos are hotter and more numerous than the neutrinos above the disk, as shown in Fig. 2, antineutrino capture on protons is favored over neutrino capture on neutrons, and the material is driven neutron rich. The neutron-to-proton ratio therefore depends in part on how many neutrino interactions take place in the outflow close to the disk. The more slowly accelerating trajectories $(\beta \geq 0.8)$ receive the greatest neutrino fluence, and, as long as the entropy is resonably high ( $s \geq 30)$, a main $r$-process results. For lower entropies, nuclei form earlier and an alpha effect, see e.g. [16], impedes the $r$ process. These trajectories, along with rapidly accelerating trajectories that are not significantly neutron rich, produce weak $r$-process nuclei.

While our results suggest that weak $r$-process nuclei are likely robustly produced in hot out- 

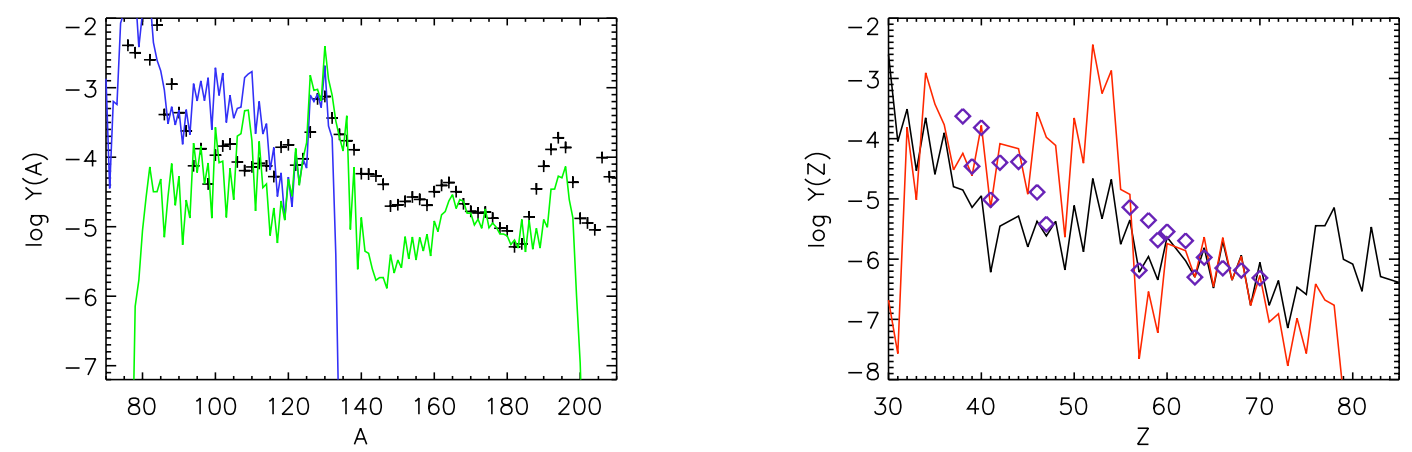

Figure 3: The left panel shows sample nucleosynthesis results as a function of mass number $A$ for outflow from the disk, compared to the scaled solar $r$-process abundances (crosses). The blue line shows a weak $r$ process from conditions, $s / k=20, \beta=0.2$, angle averaged over the disk, and the green line shows a main $r$ process from the conditions, $s / k=40, \beta=0.8$, again angle averaged around the disk. The right panel shows a sample weak $r$-process as a function of atomic number $Z$. The red line shows the calculated abundances from the disk outflow with conditions, $s / k=20, \beta=0.8$, plotted with the scaled solar $r$-process abundances (solid line) and the $r$-process abundances from metal-poor halo star HD122563 (purple diamonds).

flows from BH-NS merger accretion disks, it is unclear whether such merger events could be the sole site of the weak $r$-process. For example, if BH-NS mergers occur at a rate similar to that estimated for NS-NS mergers, $10^{-4}$ to $10^{-5}$ per year in the Galaxy [17], then $10^{-1} \mathrm{M}_{\odot}$ to $1 \mathrm{M}_{\odot}$ of weak $r$-process material would need to be ejected per merger. Current estimates of the mass ejected from merger events tends to be closer to $10^{-2} \mathrm{M}_{\odot}[18,19]$.

An additional production mechanism for compact binaries has been suggested [20] that results in the creation of tighter orbits with faster decay timescales. Should this suggestion be confirmed, BH-NS mergers may contribute to $r$-process abundances early in galactic history. Furthermore the abundance signature of an individual merger event could potentially be observed in the $r$-process pattern of a metal-poor halo star. As an example, in the right panel of Fig. 3 we plot the results from one of our trajectories against the $r$-process abundances in halo star HD122563 [21], which is known to be enriched in weak $r$-process material. The main features of this abundance pattern are well reproduced by the calculation, suggesting a possible BH-NS merger origin for the HD122563 $r$-process abundances.

\section{Conclusion}

Compact object mergers have long been thought to present attractive environments for $r$ process nucleosynthesis. Here we have examined a site within a black hole - neutron star mergerthe hot outflows from the BH-NS merger accretion disk - and found at least weak $r$-process nuclei, and perhaps main $r$-process nuclei, are likely produced in this site. While the rarity of BH-NS mergers perhaps precludes these events from being the primary weak $r$-process site, the nucleosynthetic outcome from compact object mergers should be taken into account in any galactic tally of $r$-process abundances, and potentially in the interpretation of $r$-process abundance data from individual metal-poor halo stars. 


\section{Acknowledgments}

This work was partially supported by the Department of Energy under contracts DE-FG0505ER41398 (RS) and DE-FG02-02ER41216 (GCM). This work was partially supported by the United States National Science Foundation under contract AST-0653376 (WRH). Oak Ridge National Laboratory (WRH) is managed by UT-Battelle, LLC, for the U.S. Department of Energy under contract DE-AC05-000R22725.

\section{References}

[1] E. M. Burbidge, G. R. Burbidge, W. A. Fowler, and F. Hoyle. Reviews of Modern Physics, 29:547-650, 1957.

[2] A. G. W. Cameron. Chalk River Rep., CRL-41, 1957.

[3] B. S. Meyer, G. J. Mathews, W. M. Howard, S. E. Woosley, and R. D. Hoffman. Astrophys. J., 399:656-664, November 1992.

[4] S. E. Woosley, J. R. Wilson, G. J. Mathews, R. D. Hoffman, and B. S. Meyer. Astrophys. J., 433:229-246, September 1994.

[5] K. Takahashi, J. Witti, and H.-T. Janka. Astron. Astrophys., 286:857-869, June 1994.

[6] J. Beun, G. C. McLaughlin, R. Surman, and W. R. Hix. Phys. Rev. C, 77(3):035804-+, March 2008.

[7] C. Freiburghaus, S. Rosswog, and F.-K. Thielemann. Astrophys. J., 525:L121-L124, November 1999.

[8] B. S. Meyer. Astrophys. J., 343:254-276, August 1989.

[9] R. Surman, G. C. McLaughlin, and W. R. Hix. Astrophys. J., 643:1057-1064, June 2006.

[10] S. Wanajo, M. Tamamura, N. Itoh, K. Nomoto, Y. Ishimaru, T. C. Beers, and S. Nozawa. Astrophys. J., 593:968-979, August 2003.

[11] H. Ning, Y.-Z. Qian, and B. S. Meyer. Astrophys. J., 667:L159-L162, October 2007.

[12] S. Goriely, P. Demetriou, H.-T. Janka, J. M. Pearson, and M. Samyn. Nuclear Physics A, 758:587-594, July 2005.

[13] R. Surman, G. C. McLaughlin, M. Ruffert, H.-T. Janka, and W. R. Hix. Astrophys. J., 679:L117-L120, June 2008.

[14] R. Surman and G. C. McLaughlin. Astrophys. J., 603:611-623, March 2004.

[15] R. Surman and G. C. McLaughlin. Astrophys. J., 618:397-402, January 2005.

[16] G. C. McLaughlin, G. M. Fuller, and J. R. Wilson. Astrophys. J., 472:440-+, December 1996.

[17] K. Belczynski, R. O’Shaughnessy, V. Kalogera, F. Rasio, R. E. Taam, and T. Bulik. Astrophys. J., 680:L129-L132, June 2008.

[18] M. Ruffert, H.-T. Janka, K. Takahashi, and G. Schaefer. Astron. Astrophys., 319:122-153, March 1997.

[19] R. Oechslin, H.-T. Janka, and A. Marek. Astron. Astrophys., 467:395-409, May 2007.

[20] K. Belczynski, R. Perna, T. Bulik, V. Kalogera, N. Ivanova, and D. Q. Lamb. Astrophys. J., 648:1110-1116, September 2006.

[21] S. Honda, W. Aoki, Y. Ishimaru, S. Wanajo, and S. G. Ryan. Astrophys. J., 643:1180-1189, June 2006. 